\title{
A clinical review of retinotopy
}

Jenny Nij Bijvank MD PhD ${ }^{1,2^{*}}$, Lucas Maillette de Buy Wenniger MD PhD ${ }^{2 *}$, Pim de Graaf MD PhD ${ }^{3}$, Axel Petzold PhD FRCP FRCOphth ${ }^{1,2,4}$

${ }^{1}$ Department of Neurology, MS Center and Neuro-ophthalmology Expertise Center, Amsterdam Neuroscience, Amsterdam UMC, Vrije Universiteit Amsterdam, Amsterdam, the Netherlands.

${ }^{2}$ Department of Ophthalmology, Neuro-ophthalmology Expertise Center, Amsterdam Neuroscience, Amsterdam UMC, Vrije Universiteit Amsterdam, Amsterdam, the Netherlands.

${ }^{3}$ Department of Radiology and Nuclear Medicine, Amsterdam UMC, Vrije Universiteit Amsterdam, Amsterdam, the Netherlands.

${ }^{4}$ Moorfields Eye Hospital, The National Hospital for Neurology and Neurosurgery and the UCL Queen Square Institute of Neurology, London, United Kingdom

${ }^{*}$ contributed equally

Corresponding author:

Axel Petzold

UCL \& Amsterdam UMC

a.petzold@ucl.ac.uk 


\section{Abstract}

Two observations made 29 years apart are the cornerstones of this review on the contributions of $\mathrm{Dr}$ Gordon Plant to understanding pathology affecting the optic nerve. The first observation laid the anatomical basis in 1990 for the interpretation of optical coherence tomography (OCT) findings in 2009. Retinal OCT offers clinicians detailed in vivo structural imaging of individual retinal layers. This has led to novel observations which were impossible by direct ophthalmoscopy. The technique also helps to re-introduce the anatomically grounded concept of retinotopy to clinical practise. This review illustrates the anatomical basis for retinotopy through detailed sketches (Plant \& Perry 1990). The preand post-laminar axons forming the optic nerve and their post-synaptic path from the dorsal lateral geniculate nucleus to the primary visual cortex in humans. With the mapped neuroanatomy in mind we use OCT-MRI pairs to discuss the patterns of neurodegeneration in eye and brain that are a consequence of the hard wired retinotopy: antero- and retrograde axonal degeneration which can, within the visual system, propagate trans-synaptically (Jindahra, Petrie \& Plant 2009). The technical advances of OCT and MRI for the first time enable us to trace the signals of axonal degeneration through the visual system entirely at spectacular resolution. In conclusion, the neuroanatomical insights provided by the combination of OCT and MRI allows us to separate incidental findings from sinister pathology and gives us new opportunities to tailor and monitor novel neuroprotective strategies. 


\section{Introduction}

The Japanese physician Tatsuji Inouye was first to describe the detailed associations between structural damage to the visual pathways in the human brain and the resulting visual deficit [1]. These observations and subsequent work from the Queen Square Neurologist Gordon Holmes introduced the concept of retinotopy to clinical practise [2]. The three key points relevant to this review are:

- Each point on the retina is represented in the visual occipital cortex

- The macula has the greatest area of cortical representation

- Damage to any part of this, hard-wired, pathway will cause atrophy of corresponding retinal-cortical areas.

Over the past century neurologists have, in general, not been very well trained in assessing the macula. The clinical skills of a neurologist included precise localisation of a lesion based on signs and symptoms [3]. With invention of clinically applicable brain imaging, first computed tomography (CT) [4] and then magnetic resonance imaging (MRI) [5] allowed for the visualization of brain lesions. With retinal optical coherence tomography (OCT) is has now also become possible to demonstrate the corresponding lesion in the nervous layers of the retina, including the macula [6]. The anatomical structure of the now easily accessible macula makes it a formidable area to apply retinotopy to routine clinical 
practise. In a nutshell patterns of inner retinal layer atrophy observed in the macula substitute for the well described patterns of visual field (VF) loss [7].

This review will first discuss the anatomical basis of retinotopy [8] [9] [10] [11]. This includes novel observations from the OCT literature [12; 13]. The need for a qualitative retinotopic approach to the interpretation of pattern atrophy in the macula will become evident. This will be illustrated in four own clinical cases. The review concludes with opening a window which gives a vision for future neuro-protective treatment trial design and monitoring.

\section{Anatomy}

For the past century neurologists were trained to examine the optic disc, why should the focus now be on the macula [14]? There are two anatomical reasons for this:

- there are no ganglion cells in the optic disc

- the peripapillary retinal nerve fibre layer (RNFL) projection pattern is not suitable (or practical) for strict retinotopic research because of intra-individual variation and difficulties to easily match with the visual field [15].

Figure 1 shows the anatomical relationship of the macula and optic disc [8]. The centre of the macula, called foveola, contains essentially only retinal photoreceptors in order to maximise the resolution of the central part of the visual image. In the OCT terminology, photoreceptors are summarised as the outer nuclear layer (ONL) [16]. Next, Henle fibres are very thin axons which are orientated oblique from the fovea to the perimacular rim. The perimacular rim is also called the parafoveal area and corresponds to an oval of about 0.5 $\mathrm{mm}$ in diameter. The thickness of the foveola is about $0.13 \mathrm{~mm}$ and total retinal thickness increases to about $0.5 \mathrm{~mm}$ in the perimacular rim in humans. Henle fibres are very difficult 
to see by direct ophthalmoscopy, but can be easily seen with OCT [17]. A dynamic approach is recommended and shown for teaching by video [18].

In strict hierarchical order the next neuro-axonal retinal layers in OCT terminology are the outer plexiform layer (OPL), inner nuclear layer (INL), inner plexiform layer (IPL), ganglion cell layer (GCL), retinal nerve fibre layer (RNFL) [16].

The INL is where the soma of the main glial cells of the retina, the Müller cells, are located. The role of Müller cells is to maintain the homeostasis of the retina. In retinal pathology Müller cells proliferate and upregulate glial fibrillary acidic protein (GFAP) expression [19]. Consequently, GFAP levels increase in the adjacent body fluid compartment [20]. A thickening of the INL is thought to be related to Müller cells pathology and an impaired retinal glymphatic system [21; 22]. There are physiological variations of the INL which can be demonstrated with for example a fluid challenge [23]. In contrast, thinning of the INL is not generally observed as part of the atrophy pattern following damage to the optical pathways. It was suggested that the anatomically horizontal, as well as vertical neuronal network of the INL protects the only bipolar cell of the hard wired visual pathway [24]. Because the INL acts as a "dam" ${ }^{1}$ to propagation of retrograde trans-synaptic atrophy the term, inner retinal layers will only include the next three layers discussed. This interpretation is further strengthened through meta-analysis [25]. In the inner plexiform layer (IPL) the bipolar cells of the INL, amacrine and horizontal cells all synapse with the retinal ganglion cells [26]. The area is relevant for image processing on the retinal level. Simplified, the ONL produces an analogue signal resulting from phototransduction. Next, the ribbon synapse control of the retinal bipolar cells in the INL [27]

1The term "dam" comes from the Dutch language to indicate a barrier. Embedded in the name "Amsterdam" the original description was word play "A dam" [24]. 
convert this analogue signal into digital signal [28]. These $\sim 12$ digital streams leaving the INL become $\sim 64$ digital streams, containing early visual information, once they pass through the IPL and ganglion cell layer (GCL) to reach the human brain. This is accomplished by only two axons. The first axon leaves the optic disc in a very specific retinal pathway (Figure 1) [8]. Once this non-myelinated axon leaves the retina and passes through the lamina cribrosa it becomes myelinated to contribute to the optic nerve [29] (Figure 1) [12].

Figure 1 clearly shows the horizontal architecture of the RNFL pathways. This is important because any damage occurring at level of the optic disc, as in e.g. glaucoma, will leave a horizontal pattern of RNFL atrophy in the retina and consequently a horizontal pattern of GCL atrophy in the macula. This can be memorised as "the sunset sign" [30]. Likewise, it is relevant to notice the alignment of the different retinal areas within the distal and caudal portion of the optic nerve. Located medially in the distal optic nerve, the perimacular bundle (PMB) relocates centrally in the caudal optic nerve [10]. Axons within the PMB carry the most important part of the visual image which comes from the fovea. Therefore axons of the PMB belong to the most extensively myelinated axons. This explains the selective vulnerability in demyelinating diseases [31]. Selective vulnerability may further be explained on a proteomic level as there is evidence for sectorial variation of neurofilament iso- and phosphoforms [32]. Neurofilaments (Nf) are the scaffold of the axonal cystoskeleton [33] with a wide range of functions [34]. Visualisation of early neurofilament pathology in the retina, which involves protein phosphorylation and aggregation seems tantalising near with emergence of in vivo birifrigence techniques [35], but this type of translational research has yet not materialised. 
In the retina, axons of the PMB are also the thinnest, rendering them particular vulnerable to nutritional and toxic pathologies [36] [37].

Once all retinal axons have come together to form the optic nerve, the retinotopic pattern of damage observable by OCT in the macula will be determined by the brain region within which the damage occurs (Figure 2A\&B) [12]. This is where MRI becomes important. The classical teaching example is that of damage to the optic chiasm which starts as a superior bi-temporal visual field defect (VFD) [38]. As this review will show there are specific patterns of macular GCL atrophy which correspond to the areas of the brain pathology. As a rule of thumb, lesions to the visual pathways from the chiasm onwards will leave a vertical pattern of macular GCL atrophy ("half moon sign") [30].

\section{Pathophysiology}

Nerve injury has been classified into neuropraxia, axonotomesis and neurotmesis [39]. The latter two leave a permanent deficit, whilst neuropraxia only causes conduction block with a good chance of recovery if there is no more damage. The classical example is again compression at the optic chiasm with excellent visual recovery in most cases if recognised and dealt with promptly [38]. Axonotmesis (= structural injury to axon with preservation of the surrounding connective tissue) and neurotmesis (= complete disruption of the axon and surrounding endoneurinum) compromise the axonal compartment which leads to acute release of biomarkers for neurodegeneration such as $\mathrm{Nf}$ [33] [40] [32]. Regarding the optic nerve this is followed by development of RNFL atrophy within three months [41] [42]. 
The contemporary terms for the mechanisms causing this are antero- and retrograde, trans-synaptic, axonal degeneration [12; 13] [43].

Direct anterograde axonal degeneration is also known as Wallerian degeneration. Wallerian degeneration describes what happens to the distal axonal stump. It is worth noting from August Waller's original publication that during his times there was no consensus whether this type of degeneration actually existed [44]. Two of the world experts in the field, Burdach (1837) and Valentin (1839) both stated that there is no evidence for axonal degeneration after experimental axonal injury. Living in Kent, United Kingdom at the time, Waller examined the two nerves supplying the tongue of the frog, a practical model because regional atrophy of the tong muscle can be shown. What is remarkable is not so much that Waller did excellent in choosing a good model for experimental demonstration of what came to be known as Wallerian or anterior degeneration. What is remarkable is that it took neurologists about another century to recognise that also retrograde axonal degeneration can occur. Initially termed dying back neuropathy in peripheral nervous injury, the term retrograde axonal degeneration has now become dominant. Clear experimental evidence for what concerns the very relevant transsynaptic aspect, "retrograde trans-synaptic" axonal degeneration was provided by van Buren [45]. This was 113 years after Waller's initial struggle to convince the medical community that there is indeed axonal degeneration following axonal injury. The lesson learned from this historical review is that time matters. There is a risk to overlook an effect if only examined hastily or in an ex vivo system which does not last long enough. Direct anterograde or Wallerian axonal degeneration is the quickest of all forms of axonal degeneration. Not surprisingly it was discovered first. Direct retrograde axonal degeneration takes longer [13] and in the case of trans-synaptic axonal degeneration 
within the nervous system of the human brain a time frame of up to three years has been observed [46]. Retrograde degeneration refers to the pattern of destruction that spreads backward along the axon, toward the neuron, and then into the neuron causing apoptosis.

The invention of OCT made it possible to observe retinal axons longitudinally in patients who suffered from injury to their brain $[12 ; 13]$. One model chosen to investigate this was stroke, because in these cases the onset is known [12]. The landmark paper on OCT in stroke comes from the Queen Square neurologist, Gordon Terence Plant who recognized very early the potential of OCT in establishing the concept of trans-synaptic axonal degeneration [12]. Having just moved from Bangkok (Thailand) to London (UK) around the time of the bomb attacks at 9:47 on 7 July 2005 [47], Panitha Jindahra accomplished the formidable task to prospectively collect and follow up OCTs from a cohort of deep phenotyped patients who had suffered a stroke. The thorough cross-sectional description at baseline [12] was duly followed up by presentation of longitudinal data of up to 917 days post-stroke [13]. An important co-variate recognized was age. This was considered in an equation to determine the mean peripapillary Retinal Nerve Fiber Layer thickness ( $p R N F L$ ) as follows:

mean pRNFL $(\mu \mathrm{m})=110.3-(9.08)^{\star}($ elapsed time in log years $)-(0.4)^{\star}($ age at time of measurement $)$

One criticisms of this work is that there can be no absolute certainty about timing of onset of the stroke, pre-existing strokes, relevance of vascular comorbidities and direct ischaemic insults to the LGN. The latter likely was the reason for the much faster progression of pRNFL atrophy in their patient \#11 (Figure 4 in reference [13]). In order to 
circumvent these obstacles a surgical model was chosen. Figure $2 \mathrm{~B}$ shows that following synapsing in the LGN some of the axons entering Meyers loop are located more anterior, close to the hippocampus which is the target of epilepsy surgery. A well know iatrogenic issue with surgery is neurotmensis of Meyer's loop axons [48]. Because of the interindividual variation of the exact location of these axons it is presently impossible to completely prevent this complication. For this reason visual function in individuals undergoing surgery is tested before and after surgery [49]. This can of course be elegantly combined with OCT thereby circumventing the inherent problems with stroke studies and retrograde trans-synaptic axonal degeneration [50]. Because the time point of surgery is known precisely it is much easier to review the time course of axonal degeneration. The findings are consistent with experimental data. Direct anterograde axonal degeneration is quickest and also most severe and stops at level of the $\mathrm{INL}^{2}$. Trans-synaptic retrograde axonal degeneration is slower and also stops at level of the INL. A new observation is that the size of the injury matters. A smaller brain lesion affecting less axons in Meyer's loop causes self-terminating retrograde trans-synaptic axonal degeneration within one year. A larger brain lesion causes for yet unknown reasons prolonged propagation of retrograde trans-synaptic axonal degeneration which also affects a larger area of $G C L$ in the retina.

The differences described here may at first glance seem small, even if statistically significant. Conceptually, they may however be important because they may lead to a better understanding of the neurodegenerative death cascade. The time delay of the transsynaptic part in this death cascade could open opportunities for neuroprotection.

2 This is, of course, for the case of anterior axonal degeneration originating in the outer retinal layers[43]. 


\section{Clinico-structural paradox}

The clinico-radiological paradox describes a discrepancy between the extent of abnormal signal changes on MRI and the severity of clinical symptoms [51] [52]. In analogy to the clinico-radiological paradox there appears also to be an clinico-structural paradox in the retina [53] . There are data on individuals with myelin oligodendrocyte glycoprotein (MOG) antibody disease (MOGAD) [54] that showed well preserved high contrast visual acuities (VA) even in the context of severe macular GCL and pRNFL atrophy [55] [56]. Figure 3 illustrates the clinico-structural paradox in a patient with newly diagnosed MOGAD. The retina showed rapid (4 week interval) development of severe GCL atrophy (lost $50 \%$ of layer thickness) with excellent recovery of VAs from right eye (RE) 6/60-3 to 6/6. On the left VAs were 6/5. Likewise, colour vision recovered from severe dyschromatopsia judging a red target or reading Ishihara charts $(0 / 17)$ on the right to hardly perceptible red desaturation only and reading of all 17 Ishihara charts. There was persistence of the strong relative afferent pupillary deficit (RAPD) on the right. The MRI showed enhancement of the intracranial, posterior portion of the right optic nerve as it approaches the optic chiasm. 


\section{Pitfalls with OCT}

One needs always to carefully review the raw data for the possibility of imaging artefacts [57] [58] [59]. Furthermore it is crucial to be aware of the blind spots in our understanding of the clinical relevance of several OCT image abnormalities.

- The age of the lesion and degree of axonal injury, neuropraxia/axonotomesis/neurotmesis, might influence the image.

- Gliosis can affect the retinal OCTand influence the reliability of automatic retinal layer segmentation or signal intensities.

- Rare anatomical variations may not have been described.

- The GCL could be affected by remote lesions via a retrograde downstream effect. This may for example give the false impression of a progressive optic neuropathy in patients with MS and a high MS lesion load in the posterior visual pathways.

- The OCT image could be influenced by systemic factors, e.g. metabolic, electric, enzymatic or other diffuse, transient, physiological changes.

- The likelihood for discovery of novel pathologies based on OCT findings. Two recent examples were microcystic macular oedema ${ }^{3}$ (MMO) and PHOMS [61] [62] [63] [64-66].

Our incomplete understanding of retinal OCT stresses the need for image sharing in an open access data repository, just like it was done for MRI [52].

3 We acknowledge that about in $20 \%$ of patients these microcysts are chronic, there is severe atrophy of retinal ganglion cells and the term "retrograde maculopathy" has merit [60]. 


\section{OCT helps to interpret MRI signal changes}

There is a great level of confidence interpreting a contrast enhancing optic nerve on MRI as was sown in Figure 3, in the context of a clear clinical picture. A diagnosis of acute optic neuritis $(\mathrm{ON})$ can readily be made. It is much more complex to interpret a diffuse increase of signal MRI which is non-enhancing as shown in Figure 4. In this patient the increased signal comes from an optic nerve which is reduced in diameter indicating optic atrophy. The corresponding OCT shows severe atrophy of the pRNFL, mildly cupped discs and severe atrophy of the macular GCL which did not progress over a one year observation period. His best corrected visual acuities were RE 6/9, LE 6/6 and there was no dyschromatopsia or RAPD. Intraocular pressures were high in childhood, but normalised following bilateral trabeculectomy for juvenile onset angle glaucoma. The signal increase on MRI is due to Wallerian or antero-grade axonal degeneration in an individual who has a glaucomatous optic neuropathy.

Another example is shown in Figure 5. This 29-year old patient had experienced visual difficulties especially at the end of the days for over 10 years before he presented with subnormal central vision of $20 / 30$ and $20 / 25$ and small paracentral scotomas. The OCT shows selective atrophy of the PMB. These axons are, as Figure 2A\&B illustrated, located in the optic nerve exactly so to match the MRI signal changes shown in Figure 5. A toxic cause of the chronic optic neuropathy was suspected, possibly caused by illicit drug use 
(amphetamin, GHB) or medication (SSRIs which have been described as rare causative agents), as the B vitamin tests were all normal. The loss of ganglion cells in this case could either be due to direct toxicity, or to retrograde atrophy caused by loss of optic nerve fibers.

Another situation where OCT can contribute to interpreting MRI findings is in the context of non-specific observations such as an empty sella. The historical origin of the term comes from cadaver observations showing a flattened pituitary gland with an empty appearance of the sella turcica. Rapidly adapted by radiologists with the invention of CT and MRI this term has been associated with a diagnosis of idiopathic intracranial hypertension $(\mathrm{IIH})$. The finding is however non-specific and can lead to overdiagnosis of $\mathrm{IIH}$. A number of other MRI signs for IIH have been discussed and the most specific one appears to be flattening of the posterior wall of the orbit. What the MRI does not permit to distinguish in this context is however true optic disc swelling from pseudopapilloedema. In IIH the optic discs frequently shows presence of peripapillary hyperrefective mass like structures (PHOMS) [65] [66] .

PHOMS are a novel OCT finding which was only proposed to the medical community as a term in 2018 [64]. The original description was intended to be an exclusion from true optic disc drusen (ODD) and it required a large international validation effort to agree on a distinct, feature based definition [67]. Based on this reproducible definition the presence of PHOMS was found to be frequent (62\%) in individuals with IIH [65]. The three key OCT features of PHOMS are [67]: 
i. location: strictly peripapillary and sitting on top of Bruch's membrane in the OCT Bscan. Frequently; a gap can be observed in the B-scan scans of PHOMS aligned through the centre of the optic disc;

ii. effect on adjacent retinal layers: there is typically an upward deflection (like a ski slope) of at least two of the other retinal layers;

iii. signal appearance which is similar to the reflectivity to the retinal nerve fiber and ganglion cell layers because there is evidence that they may represent axonal stasis, and because the normal egress of axons at the optic disc can resemble PHOMS. The latter is can be observed in tilted discs, discs with an elevated border and myopia. This downward slope of essentially normal axons can easily be mistaken for PHOMS.

Because it can be challenging at first to recognise these features the consortium has made 100 teaching and testing slides available as free downloadable supplementary data to the original report [67]. In clinical practise it is however much easier to scroll through a OCT volume scan and review the impression of a complete or partial torus [66]. It is suspected that the histology of PHOMS, which to date still remain to be matched to pre-enucleation OCT, will be similar to already described appearance of impaired axonal flow in a large number of species [66] [29] [66]. Of note, PHOMS are not disease specific and can be observed with a large group of pathologies or be of no further clinical significance $[65 ; 66]$.

The observation of the dynamic nature of PHOMS, which can develop de novo, increase or diminish in size $[65 ; 66]$ coincides in time with the discovery of a retinal glymphatic system [22]. Based on several lines of evidence the experimental description of a retinal glymphatic system had been anticipated for a number of years $[21 ; 68][69][70][71]$. 
There was a need to explain the physiological variation of individual retinal layers [72] [73] [23]; the dynamic nature of MMO in the majority of cases [63] [74] [75] [76]; the potential for local accumulation of neurotoxic products driving compartmentalised neurodegeneration [77]. Now that it is possible to design quantitative longitudinal studies with composite imaging modalities including OCT and MRI neuroprotective treatment strategies can be tested not only with regard to the principal action, but more importantly probably, with regard to their timing.

\section{MRI helps to interpret OCT atrophy patterns}

Unilateral brain lesions if involving the posterior visual pathway cause a contra-lateral hemianopia or quadrantanopia [12; 13] [78] [79]. The visual fields can be directly matched with the retinal OCT because by convention, and different to anything else done in clinical medicine, the right visual field is shown on the right and the left on the left. So it is as if the patient (rather then the doctor) looks down at his own fields. In contrast, in radiology everything which appears on the right of the image is truly on the left of the patient's body. This is shown for a right cerebral metastasis in Figure 6.

The pattern of a hemimacular atrophy can also be memorised as "the half moon sign" and should always at first observation lead to imaging of the brain [30].

In our experience, a lesion which only affects the posterior visual pathways does not lead to MMO. This observation is consistent with the pioneering experimental work by van Buren [45]. Whilst atrophy continues trans-synaptically causing severe inner retinal layer atrophy, MMO cannot be observed. In contrast, axonotmesis or neuropraxia affecting the 
anterior visual pathway is in a proportion of affected subjects associated with MMO [61] [63] [80] [74] [81].

\section{A vision through an open window for neuroprotective}

\section{treatment strategies}

There is proverb stating that "'When God closes a door, he opens a window".

By definition, axonotomesis and neurotmesis are like a closed door leaving a neurological deficit behind. But at the time axonotomesis and neurotmesis occur there may still be a chance to salvage the post-synaptic downstream effects. This time interval may be substantial because of the slow propagation of trans-synaptic degeneration which can take over one year as reviewed here.

This observation may be particular relevant in the context of larger lesions to the human brain because in addition to a trans-synaptically ruled pathway of neurodegeneration [12; 13] there is evidence for special spatial stoichiometric relationship of the axonoligodendrocyte interface [82]. Axons are electrically and metabolically isolated [83] which renders them vulnerable to a failing support line. The prolonged and spreading retinal atrophy after substantial damage to Meyer's loop in epilepsy surgery opens up a window of opportunity to halt the trans-synaptic part of neurodegeneration [49], in which case OCT imaging will be the ideal modality to monitor treatment effects. The aim of such future neuroprotective treatment strategies would not be to reverse what nature has taken, but to help the human body in what it is good: healing what is damaged. 


\section{Figure legends}

Figure 1: "The anatomical basis of the caecocentral scotoma." (A) The photomicrograph shows the axons projecting from the retinal ganglion cells of the foveola to the optic disc in a flat mounted retina of $M$. mulatta. (B) A sketch of individual retinal ganglion cells and their axons. This illustrates that all axons tend to avoid the horizontal meridian. Once these axons reach the lamina cribrosa they become myelinated and form the optic nerve which projects to the dorsal lateral geniculate nucleus (DGN) of the thalamus. This anatomical relationships are relevant for interpretation of the OCT findings because damage anywhere in this pathway will cause axonal degeneration in both directions (antero- and retrograde) resulting
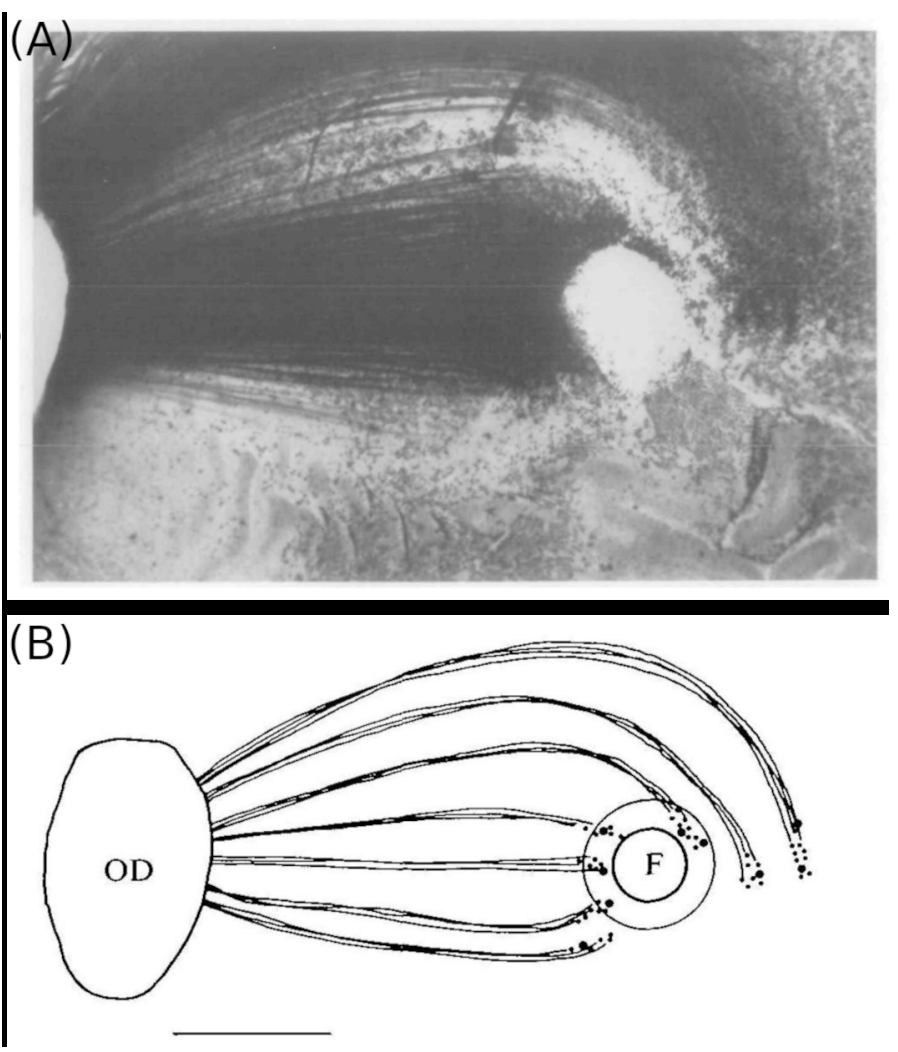
in characteristic OCT atrophy patterns, which if attitudinal indicate pathology involving the optic disc (Figures reprinted with permission from [8]). 
Figure 2: Anatomical sketch of the retinal glanglion cell axonal pathway pattern from the retina through the chiasm. This anatomical relationship is relevant for interpretation of the OCT atrophy findings resulting from retrograde trans-synaptic axonal degeneration. The MRI shows in most cases the site of pathology. In a very small proportion the lesion can be below the resolution of the MRI or are missed because of a number of other

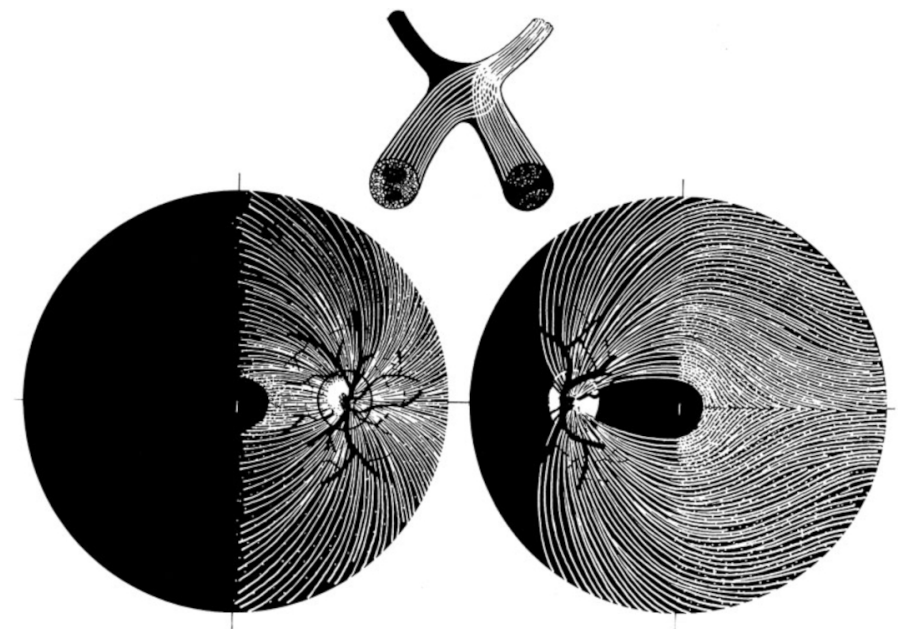
reasons known as the clinico-radiological paradox (Figures reprinted with permission from [12]). 
Figure 3: OCT and MRI from an individual with MOG-ON. Inner retinal layer atrophy is due to direct, retrograde axonal degeneration. This is severe and rapid. MRI in the acute phase shows a contrast enhancing optic nerve.

Figure 4: OCT and MRI from an individual with bilateral glaucoma. Bilateral inner retinal layer atrophy is severe and the cause of anterograde axonal degeneration. This results in an increased signal of the optic nerve on MRI which is not contrast enhancing.
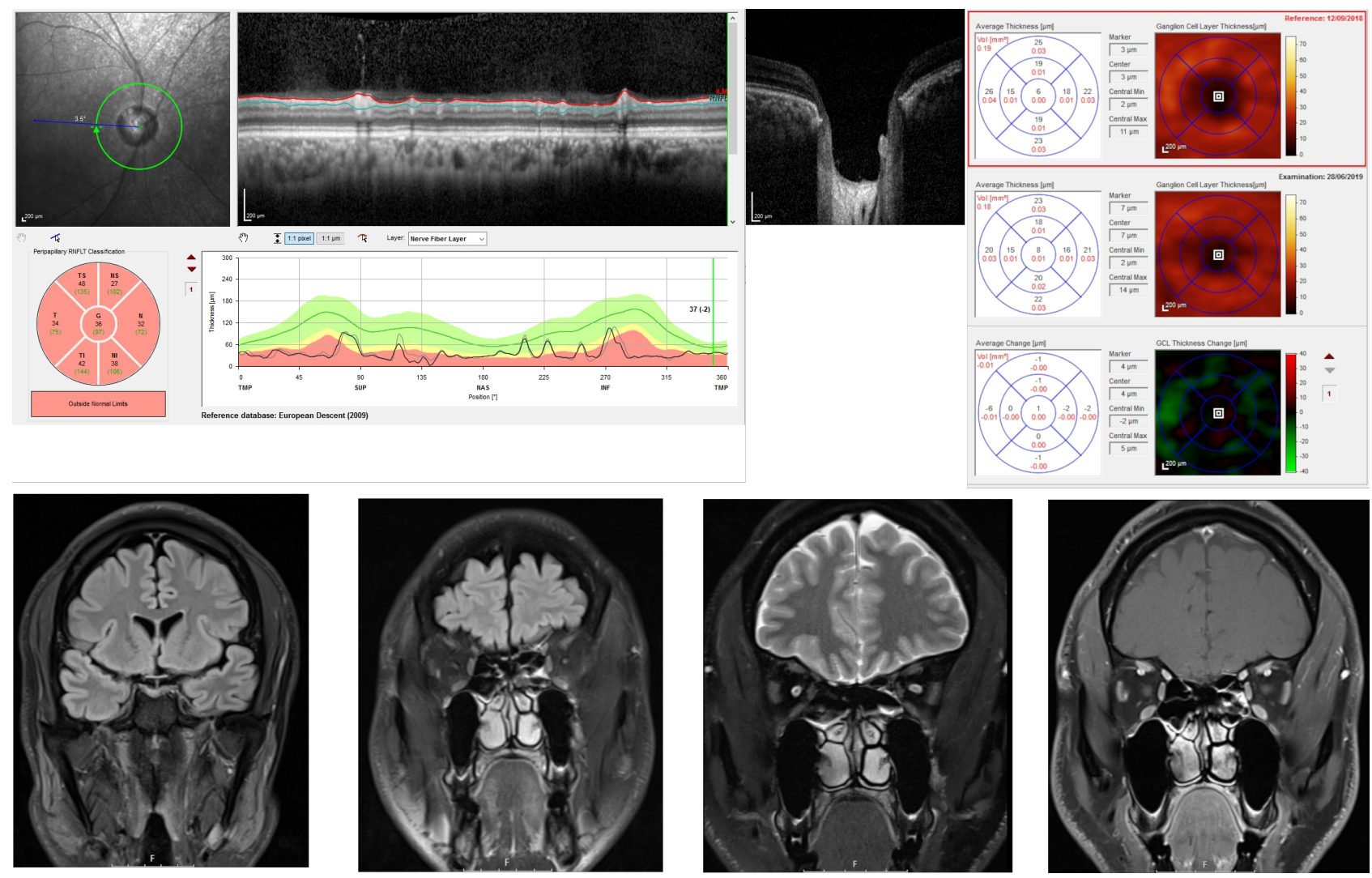
Figure 5: OCT and MRI from an 29-year old individual with a suspected toxic optic neuropathy which shows selective atrophy of the PMB on OCT. The resulting anterograde axonal degeneration gives bright (white dot inside the optic nerve in this image) signal changes on the MRI (coronal STIR) along the course of the PMB in both optic nerves. The atrophy of the caeco-central projections as beautifully demonstrated in Figure $2 A \& B$ are visible on the heat map of the RNFL. The OCT composite image shows the loss of these axons as a dark area between the optic disc and fovea. Superiorly and inferiorily to the optic disc there are still axons left showing in the heat map as a bright, yellowish area. The $6 \mathrm{~mm}$ EDTRS grid (blue) demonstrates that this loss of axons encompasses almost the entire macula. The superimposed (green coloured) heat map of the macular ganglion cell layer reveals more profound ganglion cell loss in the more severely denervated area of the macula (similar to the scattered dots in the sketch in Figure 2B). The B-scans demonstrate the lines of retinal layer segmentation for the RNFL (red and green lines) through the foveola and at the optic disc; and for the ganglion cell layer (green and purple lines) through the foveola. The findings of the composite volume scans are consistent with those of the ringscan demonstrating visually and in numbers the isolated atrophy of the papillomacular bundle (PMB).

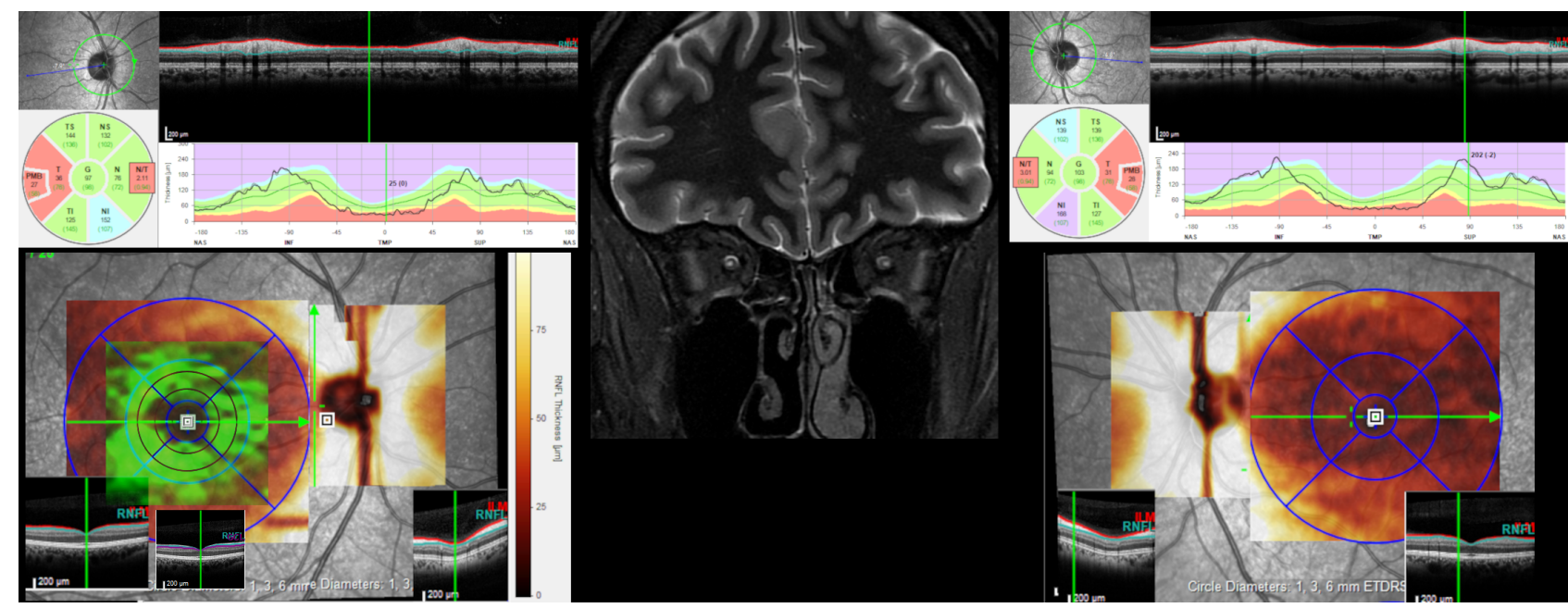


Figure 6: OCT and MRI from an individual with brain metastases of a non-small cell lung adenocarcinoma and a large occipital lesion on the right side, which causes anatomically corresponding (see sketch in Figure 2) inner macular GCL atrophy in both eyes. If observed in clinic this pattern of atrophy, which can be memorised as "the half moon sign" should
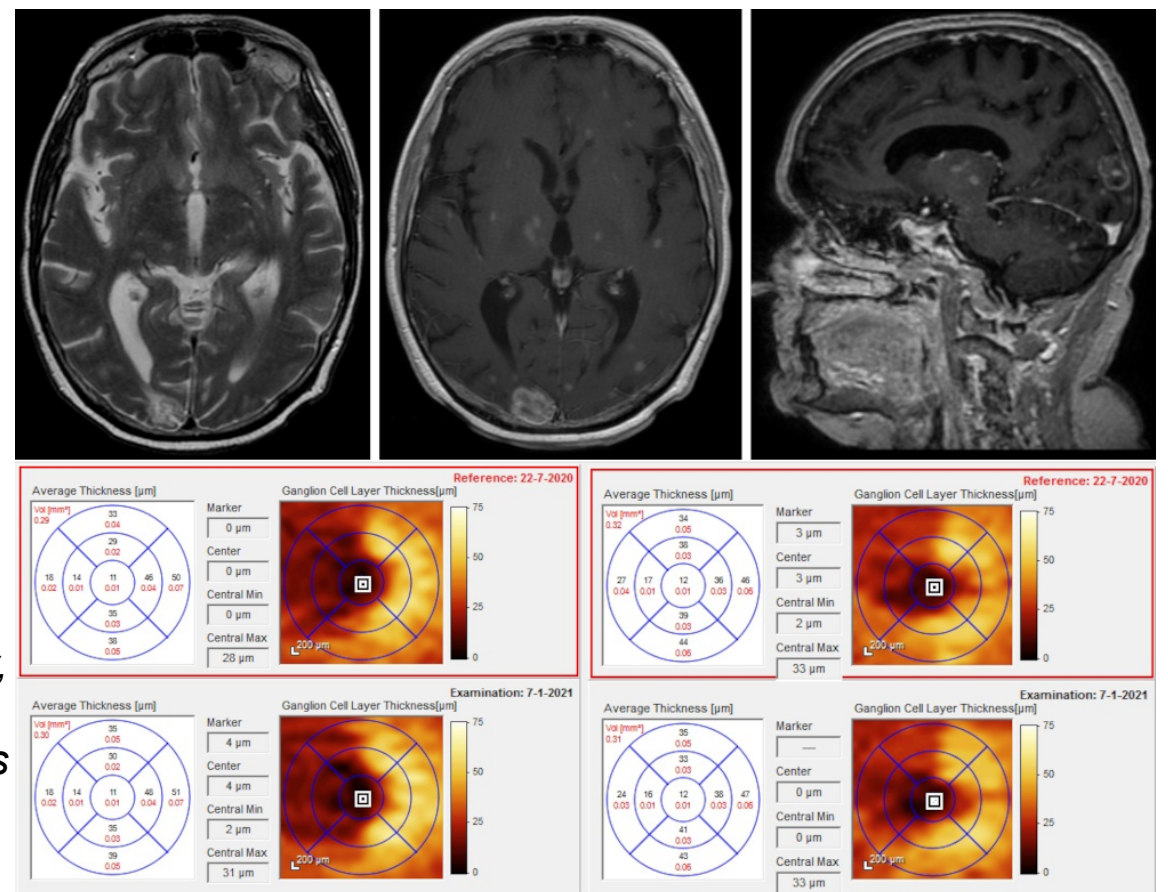

always trigger imaging of the brain. In this 72-year old patient the central vision was 20/30 in both eyes, but the visual field test showed a deep but incomplete homonymous hemianopsy of the left visual field. 


\section{References}

[1] Inouye, T., 1909. Die Sehstörungen bei Schußverletzungen der kortikalen Sehsphäre. Verlag von Wilhelm Engelsmann (Leipzig), .

[2] Holmes, G. (1945). Ferrier Lecture: The organization of the visual cortex in man, Proceedings of the Royal Society of London. Series B, Biological Sciences 132 : 348-361.

[3] Hickman, S. J. (2011). Neuro-ophthalmology., Pract Neurol 11 : 191-200.

[4] Hounsfield, G. N. (1976). Historical notes on computerized axial tomography., Journal of the Canadian Association of Radiologists 27 : 135-42.

[5] Stehling, M.; Turner, R. and Mansfield, P. (1991). Echo-planar imaging: magnetic resonance imaging in a fraction of a second, Science $254: 43-50$.

[6] Huang, D.; Swanson, E.; Lin, C.; Schuman, J.; Stinson, W.; Chang, W.; Hee, M.; Flotte, T.; Gregory, K.; Puliafito, C. and al. et (1991). Optical coherence tomography., Science 254 : 1178-1181.

[7] Costello, F. and Scott, J. N. (2019). Imaging in Neuro-ophthalmology, CONTINUUM: Lifelong Learning in Neurology 25 : 1438-1490.

[8] Plant, G. T. and Perry, V. H. (1990). The anatomical basis of the caecocentral scotoma. New observations and a review., Brain 113 ( Pt 5) : 1441-1457.

[9] Aine, C. J.; Supek, S.; George, J. S.; Ranken, D.; Lewine, J.; Sanders, J.; Best, E.; Tiee, W.; Flynn, E. R. and Wood, C. C. (1996). Retinotopic Organization of Human Visual Cortex: Departures from the Classical Model, Cerebral Cortex 6 : 354-361.

[10] Fitzgibbon, T. and Taylor, S. (1996). Retinotopy of the human retinal nerve fibre layer and optic nerve head, The Journal of Comparative Neurology $375: 238-251$.

[11] Tootell, R. B. H.; Hadjikhani, N. K.; Vanduffel, W.; Liu, A. K.; Mendola, J. D.; Sereno, M. I. and Dale, A. M. (1998). Functional analysis of primary visual cortex (V1) in humans, Proceedings of the National Academy of Sciences $95: 811-817$.

[12] Jindahra, P.; Petrie, A. and Plant, G. T. (2009). Retrograde trans-synaptic retinal ganglion cell loss identified by optical coherence tomography., Brain 132 : 628-634.

[13] Jindahra, P.; Petrie, A. and Plant, G. T. (2012). The time course of retrograde transsynaptic degeneration following occipital lobe damage in humans., Brain 135 : 534-541.

[14] Micieli, J. A.; Newman, N. J. and Biousse, V. (2019). The role of optical coherence tomography in the evaluation of compressive optic neuropathies, Current Opinion in Neurology 32 : 115-123. 
[15] Zangerl, B.; Whatham, A.; Kim, J.; Choi, A.; Assaad, N. N.; Hennessy, M. P. and Kalloniatis, M. (2016). Reconciling visual field defects and retinal nerve fibre layer asymmetric patterns in retrograde degeneration: an extended case series., Clinical \& experimental optometry .

[16] Cruz-Herranz, A.; Balk, L. J.; Oberwahrenbrock, T.; Saidha, S.; Martinez-Lapiscina, E. H.; Lagreze, W. A.; Schuman, J. S.; Villoslada, P.; Calabresi, P.; Balcer, L.; Petzold, A.; Green, A. J.; Paul, F.; Brandt, A. U.; Albrecht, P. and consortium , I. (2016). The APOSTEL recommendations for reporting quantitative optical coherence tomography studies., Neurology 86 : 2303-2309.

[17] Otani, T.; Yamaguchi, Y. and Kishi, S. (2011). Improved visualization of Henle fiber layer by changing the measurement beam angle on optical coherence tomography., Retina $31: 497-501$.

[18] Balk, L. J.; de Vries-Knoppert, W. A. E. J. and Petzold, A. (2012). A Simple Sign for Recognizing Off-Axis OCT Measurement Beam Placement in the Context of Multicentre Studies., PLoS One 7 : e48222.

[19] Dyer, M. A. and Cepko, C. L. (2000). Control of Müller glial cell proliferation and activation following retinal injury, Nature Neuroscience 3 : 873-880.

[20] Jünemann, A. G. M.; Rejdak, R.; Huchzermeyer, C.; Maciejewski, R.; Grieb, P.; Kruse, F. E.; Zrenner, E.; Rejdak, K. and Petzold, A. (2015). Elevated vitreous body glial fibrillary acidic protein in retinal diseases., Graefes Arch Clin Exp Ophthalmol .

[21] Petzold, A. (2016). Retinal glymphatic system: an explanation for transient retinal layer volume changes? Brain $139:$ 2816-2819.

[22] Wang, X.; Lou, N.; Eberhardt, A.; Yang, Y.; Kusk, P.; Xu, Q.; Förstera, B.; Peng, S.; Shi, M.; de-Guevara, A. L.; Delle, C.; Sigurdsson, B.; Xavier, A. L. R.; Ertürk, A.; Libby, R. T.; Chen, L.; Thrane, A. S. and Nedergaard, M. (2020). An ocular glymphatic clearance system removes $\beta$-amyloid from the rodent eye, Science Translational Medicine 12 : eaaw3210.

[23] Balk, L. J.; Oberwahrenbrock, T.; Uitdehaag, B. M. and Petzold, A. (2014). Physiological variation of retinal layer thickness is not caused by hydration: $A$ randomised trial., Journal of the Neurological Sciences $344: 88-93$.

[24] Balk, L. J.; Twisk, J. W. R.; Steenwijk, M. D.; Daams, M.; Tewarie, P.; Killestein, J.; Uitdehaag, B. M. J.; Polman, C. H. and Petzold, A. (2014). A dam for retrograde axonal degeneration in multiple sclerosis?, Journal of Neurology, Neurosurgery \& Psychiatry 85 : 782-789.

[25] Petzold, A.; Balcer, L. J.; Calabresi, P. A.; Costello, F.; Frohman, T. C.; Frohman, E. M.; Martinez-Lapiscina, E. H.; Green, A. J.; Kardon, R.; Outteryck, O.; Paul, F.; Schippling, S.; Vermersch, P.; Villoslada, P.; Balk, L. J. and IMSVISUAL, E.-E. (2017). Retinal layer 
segmentation in multiple sclerosis: a systematic review and meta-analysis., The Lancet Neurology 16 : 797-812.

[26] Evangelou, N. and Alrawashdeh, O. S. M. (2016). Anatomy of the Retina and the Optic Nerve. In: (Ed.), Optical Coherence Tomography in Multiple Sclerosis, Springer International Publishing.

[27] James, B.; Darnet, L.; Moya-Daz, J.; Seibel, S.-H. and Lagnado, L. (2019). An amplitude code transmits information at a visual synapse, Nature Neuroscience 22 : 11401147.

[28] Masland, R. H. (2012). The neuronal organization of the retina., Neuron 76 : 266-280.

[29] Hollander, H.; Makarov, F.; Stefani, F. and Stone, J. (1995). Evidence of Constriction of Optic Nerve Axons at the Lamina cribrosa in the Normotensive Eye in Humans and Other Mammals, Ophthalmic Research 27 : 296-309.

[30] Petzold, A. (2021). Three 'Red Lines' for Pattern Recognition-Based Differential Diagnosis Using Optical Coherence Tomography in Clinical Practice, Journal of NeuroOphthalmology : 385-398.

[31] Uhthoff, W. (1890). Untersuchungen über die bei der mutliplen Herdsklerose vorkommenden Augensehstörungen, Arch Psychiatr Nervenkrankh 21 : 55-116,303-410.

[32] Kang, M. H.; Law-Davis, S.; Balaratnasingam, C. and Yu, D.-Y. (2014). Sectoral variations in the distribution of axonal cytoskeleton proteins in the human optic nerve head, Experimental Eye Research 128 : 141-150.

[33] Petzold, A. (2005). Neurofilament phosphoforms: Surrogate markers for axonal injury, degeneration and loss, Journal of the Neurological Sciences 233 : 183-198.

[34] Shea, T. B. and Lee, S. (2012). The discontinuous nature of neurofilament transport accommodates both establishment and repair of the axonal neurofilament array., Cytoskeleton (Hoboken) .

[35] Petzold, A.; de Boer, J. F.; Schippling, S.; Vermersch, P.; Kardon, R.; Green, A.; Calabresi, P. A. and Polman, C. (2010). Optical coherence tomography in multiple sclerosis: a systematic review and meta-analysis., The Lancet Neurol 9 : 921-932.

[36] Kisimbi, J.; Shalchi, Z.; Mahroo, O. A.; Mhina, C.; Sanyiwa, A. J.; Mabey, D.; Mohamed, M. and Plant, G. T. (2013). Macular spectral domain optical coherence tomography findings in Tanzanian endemic optic neuropathy, Brain 136 : 3418-3426.

[37] Uhthoff, W. (1880). Beitrag zur Sehnervenatrophie, Archiv für Ophthalmologie 26 : 244-282.

[38] Petzold, A. and Plant, G.T. (2001). Failure to detect bitemporal field defects due to chiasmal compression on a screening perimetry protocol, Neuro Ophthalmol 24 : 357-361. 
[39] Seddon, H. (1942). A classification of nerve injuries, British medical journal 2 : 237.

[40] Khalil, M.; Teunissen, C. E.; Otto, M.; Piehl, F.; Sormani, M. P.; Gattringer, T.; Barro, C.; Kappos, L.; Comabella, M.; Fazekas, F.; Petzold, A.; Blennow, K.; Zetterberg, H. and Kuhle, J. (2018). Neurofilaments as biomarkers in neurological disorders., Nature Reviews Neurology 14 : 577-589.

[41] Petzold, A.; Rejdak, K. and Plant, G. T. (2004). Axonal degeneration and inflammation in acute optic neuritis, J Neurol Neurosurg Psychiatry 75 : 1178-1180.

[42] Bsteh, G.; Berek, K.; Hegen, H.; Teuchner, B.; Buchmann, A.; Voortman, M. M.; Auer, M.; Wurth, S.; Zinganell, A.; Pauli, F. D.; Deisenhammer, F.; Khalil, M. and Berger, T. (2019). Serum neurofilament levels correlate with retinal nerve fiber layer thinning in multiple sclerosis., Multiple sclerosis (Houndmills, Basingstoke, England) 26 : 1682-1690.

[43] Panneman, E.; Coric, D.; Tran, L.; de Vries-Knoppert, W. and Petzold, A. (2019). Progression of Anterograde Trans-Synaptic Degeneration in the Human Retina Is Modulated by Axonal Convergence and Divergence, Neuro-Ophthalmology 43 : 382-390.

[44] Waller, A. (1850). Experiments on the section of glossopharyngeal and hypoglossal nerves of the frog and observations of the alternatives produced thereby in the structure of their primitive fibres, Philos Trans R Soc London 140 : 423-429.

[45] van Buren, J. (1963). Trans-synaptic retrograde degeneration in the visual system of primates, J Neurol Neurosurg Neuropsych 26 : 402-409.

[46] Petzold, A. (2014). Neurodegeneration and Multiple Sclerosis. In: (Ed.), Neurodegenerative Diseases, Springer London.

[47] Tisdall, M. and Petzold, A. (2012). Comment on "Chronic Traumatic Encephalopathy in Blast-Exposed Military Veterans and a Blast Neurotrauma Mouse Model", Science Translational Medicine 4 : 157le8; author reply 157Ir5.

[48] Jeelani, N. U. O.; Jindahra, P.; Tamber, M. S.; Poon, T. L.; Kabasele, P.; JamesGalton, M.; Stevens, J.; Duncan, J.; McEvoy, A. W.; Harkness, W. and Plant, G. T. (2010). emispherical asymmetry in the Meyerś Loop a prospective study of visual-field deficits in 105 cases undergoing anterior temporal lobe resection for epilepsy., J Neurol Neurosurg Psychiatry 81 : 985-991.

[49] de Vries-Knoppert, W. A.; Baaijen, J. C. and Petzold, A. (2019). Patterns of retrograde axonal degeneration in the visual system, Brain $142: 2775-2786$.

[50] Pula, J. H. and Yuen, C. A. (2017). Eyes and stroke: the visual aspects of cerebrovascular disease, Stroke and Vascular Neurology 2 : 210-220.

[51] Fouad, K.; Popovich, P. G.; Kopp, M. A. and Schwab, J. M. (2020). The neuroanatomical-functional paradox in spinal cord injury, Nature Reviews Neurology 17 : 53-62. 
[52] Bijvank, J. A. N.; Aliaga, E. S.; Balk, L. J.; Coric, D.; Davagnanam, I.; Tan, H. S.; Uitdehaag, B. M. J.; Rijn, L. J. and Petzold, A. (2021). A model for interrogating the clinicoradiological paradox in multiple sclerosis: Internuclear ophthalmoplegia, European Journal of Neurology 28 : 1617-1626.

[53] Meneguette, N. S.; Almeida, K. M. F. R.; de Oliveira Figueiredo, M. T. J.; de Araújo e Araújo, A. C. R.; Alvarenga, M. P.; Vasconcelos, C. C. F.; Nascimento, A. C. B.; Colombini, G. N. U. I.; Petzold, A. and Alvarenga, R. M. P. (2021). Optic neuritis in Asian type opticospinal multiple sclerosis (OSMS-ON) in a non-Asian population: a functionalstructural paradox, Multiple Sclerosis and Related Disorders : 103260.

[54] Marignier, R.; Hacohen, Y.; Cobo-Calvo, A.; Pröbstel, A.-K.; Aktas, O.; Alexopoulos, H.; Amato, M.-P.; Asgari, N.; Banwell, B.; Bennett, J.; Brilot, F.; Capobianco, M.; Chitnis, T.; Ciccarelli, O.; Deiva, K.; Sèze, J. D.; Fujihara, K.; Jacob, A.; Kim, H. J.; Kleiter, I.; Lassmann, H.; Leite, M.-I.; Linington, C.; Meinl, E.; Palace, J.; Paul, F.; Petzold, A.; Pittock, S.; Reindl, M.; Sato, D. K.; Selmaj, K.; Siva, A.; Stankoff, B.; Tintore, M.; Traboulsee, A.; Waters, P.; Waubant, E.; Weinshenker, B.; Derfuss, T.; Vukusic, S. and Hemmer, B. (2021). Myelin-oligodendrocyte glycoprotein antibody-associated disease, The Lancet Neurology $20: 762-772$.

[55] Petzold, A.; Wong, S. and Plant, G. T. (2016). Autoimmunity in visual loss., Handb Clin Neurol $133:$ 353-376.

[56] Ciron, J.; Cobo-Calvo, A.; Audoin, B.; Bourre, B.; Brassat, D.; Cohen, M.; Collongues, N.; Deschamps, R.; Durand-Dubief, F.; Laplaud, D.; Maillart, E.; Papeix, C.; Zephir, H.; Bereau, M.; Brochet, B.; Carra-Dallière, C.; Derache, N.; Gagou-Scherer, C.; Henry, C.; Kerschen, P.; Mathey, G.; Maubeuge, N.; Maurousset, A.; Montcuquet, A.; Moreau, T.; Prat, C.; Taithe, F.; Thouvenot, E.; Tourbah, A.; Rollot, F.; Vukusic, S. and Marignier, R. (2019). Frequency and characteristics of short versus longitudinally extensive myelitis in adults with MOG antibodies: A retrospective multicentric study, Multiple Sclerosis Journal 26 : 936-944.

[57] Tewarie, P.; Balk, L.; Costello, F.; Green, A.; Martin, R.; Schippling, S. and Petzold, A. (2012). The OSCAR-IB Consensus Criteria for Retinal OCT Quality Assessment., PLoS ONE 7 : e34823.

[58] Schippling, S.; Balk, L.; Costello, F.; Albrecht, P.; Balcer, L.; Calabresi, P.; Frederiksen, J.; Frohman, E.; Green, A.; Klistorner, A.; Outteryck, O.; Paul, F.; Plant, G.; Traber, G.; Vermersch, P.; Villoslada, P.; Wolf, S. and Petzold, A. (2015). Quality control for retinal OCT in multiple sclerosis: validation of the OSCAR-IB criteria., Mult Scler 21 : 163-170.

[59] Oberwahrenbrock, T.; Weinhold, M.; Mikolajczak, J.; Zimmermann, H.; Paul, F.; Beckers, I. and Brandt, A. U. (2015). Reliability of Intra-Retinal Layer Thickness Estimates., PLoS One 10 : e0137316. 
[60] Mahroo, O. A.; Shalchi, Z.; Kisimbi, J.; Sanyiwa, A. J.; Mohamed, M. D. and Plant, G. T. (2014). Re: Abegg et al.: Microcystic Macular Edema: Retrograde Maculopathy Caused by Optic Neuropathy (Ophthalmology 2014;121:142-9)., Ophthalmology 121 : e40.

[61] Gelfand, J. M.; Nolan, R.; Schwartz, D. M.; Graves, J. and Green, A. J. (2012). Microcystic macular oedema in multiple sclerosis is associated with disease severity., Brain $135: 1786-1793$.

[62] Petzold, A. (2012). Microcystic macular oedema in MS: T2 lesion or black hole?, Lancet Neurol 11 : 933-934.

[63] Burggraaff, M. C.; Trieu, J.; de Vries-Knoppert, W. A. E. J.; Balk, L. and Petzold, A. (2014). The Clinical Spectrum of Microcystic Macular Edema, Investigative Opthalmology \& Visual Science $55:$ 952-961.

[64] Malmqvist, L.; Bursztyn, L.; Costello, F.; Digre, K.; Fraser, J. A.; Fraser, C.; Katz, B.; Lawlor, M.; Petzold, A.; Sibony, P.; Warner, J.; Wegener, M.; Wong, S. and Hamann, S. (2018). The Optic Disc Drusen Studies Consortium Recommendations for Diagnosis of Optic Disc Drusen Using Optical Coherence Tomography, Journal of NeuroOphthalmology $38: 299-307$.

[65] Petzold, A.; Coric, D.; Balk, L. J.; Hamann, S.; Uitdehaag, B. M. J.; Denniston, A. K.; Keane, P. A. and Crabb, D. P. (2020). Longitudinal Development of Peripapillary HyperReflective Ovoid Masslike Structures Suggests a Novel Pathological Pathway in Multiple Sclerosis, Annals of Neurology 88 : 309-319.

[66] Fraser, J. A.; Sibony, P. A.; Petzold, A.; Thaung, C. and Hamann, S. (2021). Peripapillary Hyper-reflective Ovoid Mass-like Structure (PHOMS), Journal of NeuroOphthalmology Publish Ahead of Print.

[67] Petzold, A.; Biousse, V.; Bursztyn, L.; Costello, F.; Crum, A.; Digre, K.; Fraser, C.; Fraser, J. A.; Katz, B.; Jurkute, N.; Newman, N.; Lautrup-Battistini, J.; Lawlor, M.; Liskova, P.; Lorenz, B.; Malmqvist, L.; Peragallo, J.; Sibony, P.; Subramanian, P.; Rejdak, R.; Nowomiejska, K.; Touitou, V.; Warner, J.; Wegener, M.; Wong, S.; Wai-Man, P. Y. and Hamann, S. (2020). Multirater Validation of Peripapillary Hyperreflective Ovoid Mass-like Structures (PHOMS), Neuro-Ophthalmology 44 : 413-414.

[68] Wostyn, P.; De Groot, V.; Van Dam, D.; Audenaert, K.; Killer, H. E. and De Deyn, P. P. (2016). Age-related macular degeneration, glaucoma and Alzheimer's disease: amyloidogenic diseases with the same glymphatic background?, Cellular and Molecular Life Sciences 73 : 4299-4301.

[69] Mathieu, E.; Gupta, N.; Ahari, A.; Zhou, X.; Hanna, J. and Yücel, Y. H. (2017). Evidence for Cerebrospinal Fluid Entry Into the Optic Nerve via a Glymphatic Pathway, Investigative ophthalmology \& visual science 58 : 4784-4791. 
[70] Lenck, S.; Radovanovic, I.; Nicholson, P.; Hodaie, M.; Krings, T. and Mendes-Pereira, V. (2018). Idiopathic intracranial hypertension: The veno glymphatic connections., Neurology $91: 515-522$.

[71] Denniston, A. K. and Keane, P. A. (2016). Black Holes and the Ocular Glymphatic System: Author Response to The Glymphatic System: A New Player in Ocular Diseases? Letters, Investigative ophthalmology \& visual science 57 : 5428-5428.

[72] Balk, L. J.; Sonder, J. M.; Strijbis, E. M. M.; Twisk, J. W. R.; Killestein, J.; Uitdehaag, B. M. J.; Polman, C. H. and Petzold, A. (2012). The physiological variation of the retinal nerve fiber layer thickness and macular volume in humans as assessed by spectral domain-optical coherence tomography., Invest Ophthalmol Vis Sci 53 : 1251-1257.

[73] Balk, L.; Mayer, M.; Uitdehaag, B. and Petzold, A. (2013). Physiological variation of segmented OCT retinal layer thicknesses is short-lasting., J Neurol 260 : 3109-3114.

[74] Saidha, S.; Sotirchos, E. S.; Ibrahim, M. A.; Crainiceanu, C. M.; Gelfand, J. M.; Sepah, Y. J.; Ratchford, J. N.; Oh, J.; Seigo, M. A.; Newsome, S. D.; Balcer, L. J.; Frohman, E. M.; Green, A. J.; Nguyen, Q. D. and Calabresi, P. A. (2012). Microcystic macular oedema, thickness of the inner nuclear layer of the retina, and disease characteristics in multiple sclerosis: a retrospective study., The Lancet Neurology 11 : 963-972.

[75] Borruat, F.-X.; Dysli, M.; Voide, N. and Abegg, M. (2018). Acetazolamide Reduces Retinal Inner Nuclear Layer Thickness in Microcystic Macular Edema Secondary to Optic Neuropathy, European Neurology 79 : 150-153.

[76] Knier, B.; Schmidt, P.; Aly, L.; Buck, D.; Berthele, A.; Mühlau, M.; Zimmer, C.; Hemmer, B. and Korn, T. (2016). Retinal inner nuclear layer volume reflects response to immunotherapy in multiple sclerosis., Brain 139 : 2855-2863.

[77] Wostyn, P. (2020). The ocular glymphatic clearance system: a key missing piece of the Alzheimer's disease-glaucoma puzzle found?, Eye .

[78] Petzold, A. and Plant, G. (2005). Central and paracentral visual field defects and driving abilities, Ophthalmologica 219 : 191-201.

[79] Horton, J. and Hoyt, W. (1991). Quadrantic visual field defects. A hallmark of lesions in extrastriate (V2/V3) cortex, Brain 114 : 1703-1718.

[80] Abegg, M.; Dysli, M.; Wolf, S.; Kowal, J.; Dufour, P. and Zinkernagel, M. (2014). Microcystic macular edema: retrograde maculopathy caused by optic neuropathy., Ophthalmology $121: 142-149$.

[81] Pott, J. W. R.; de Vries-Knoppert, W. A. E. J. and Petzold, A. (2016). The prevalence of microcystic macular changes on optical coherence tomography of the macular region in optic nerve atrophy of non-neuritis origin: a prospective study., The British journal of ophthalmology $100: 216-221$. 
[82] Simons, M.; Misgeld, T. and Kerschensteiner, M. (2014). A unified cell biological perspective on axon-myelin injury., J Cell Biol 206 : 335-345.

[83] Nave, K.-A. (2010). Myelination and the trophic support of long axons, Nature Reviews Neuroscience $11: 275-283$. 


\section{Disclosures}

-J.A. Nij Bijvank is supported by the Dutch MS Research Foundation, grant nr. 18-1027 and servers on the Editorial Board of Neuro-ophthalmology.

- L. Maillette de Buy Wenniger has no conflicts of interest to report.

- P de Graaf has no conflicts of interest to report.

-A. Petzold reports speaker fees from Heidelberg Engineering, outside the submitted work; and is part of the steering committee of the OCTiMS study which is sponsored by Novartis and the OCTA Angie and Ari steering committees which are sponsored by Zeiss. He does not receive compensation for these activities

\section{Funding Statement}

This research received no specific grant from any funding agency in the public, commercial or not-for-profit sectors.

\section{Author contributions}

Writing of first draft of invited review: AP; contribution of intellectual content, clinical content and figures JANB, LMdBW, PdG. Revision of text, all authors.

\section{Acknowledgement}

The review was presented as part of a Festschrift to honour the contributions of $\mathrm{Dr}$

Gordon Terence Plant to neuro-ophthalmology and to thank for his mentorship ever since first meeting at the XII. Symposium of the International Research Group for Color Vision Deficiencies in Tübingen, Germany in July 1993. 